\title{
Beiträge zur Kenntniss des Biguanids.
}

\author{
Von Friedrieh Emich. \\ (Aus dem Laboratorium des Prof. R. Maly in Graz.)
}

(Vorgelegt in der sitzung am 4. Mai 1883.)

Die vorliegende Arbeit hat zunächst den Zweck, einige Angaben, welche Rathke ${ }^{1}$ iber die interessante Base, das Biguanid $\mathrm{C}_{2} \mathrm{H}_{7} \mathrm{~N}_{5}$, gemacht hat, richtig zu stellen, namentlich jene, die sich auf das Sättigungsvermögen gegen Säuren und die angebliche Änderung dieses Sättigungsvermögens durch den Eintritt von Kupfer beziehen, wobei bekanntlich eine neue Base, das rothe Biguanidkupfer $\mathrm{C}_{2} \mathrm{H}_{6}$ cu $\mathrm{N}_{5}$ entsteht.

Der zweite Theil der Arbeit enthält einen Beitrag zur Constitution des Biguanids, das, wie ich gefunden, unter dem Einflusse von verdunnter Schwefelsäure bei höherer Temperatur unter Wasseraufnahme glatt in Kohlensäure und Ammoniak gespalten wird.

I.

Rathke l.c. gibt an, dass das Biguanid $\mathrm{C}_{2} \mathrm{H}_{7} \mathrm{~N}_{5}$ zwei Moleküle einbasischer Säuren oder ein Molekul der zweibasischen Schwefelsäure zul neutral reagirenden Salzen binde, und er beschreibt genauer das schwefelsaure Biguanid fur welches er die Zusammensetzung $\mathrm{C}_{2} \mathrm{H}_{7} \mathrm{~N}_{5} \cdot \mathrm{H}_{2} \mathrm{SO}_{4}+\mathrm{H}_{2} \mathrm{O}$ findet.

Die Zusammensetzung des Sulfats vom Biguanidkupfer hat Rathke aleer $\left(\mathrm{C}_{2} \mathrm{H}_{6} \text { cu } \mathrm{N}_{5}\right)_{2} \cdot \mathrm{H}_{2} \mathrm{SO}_{4}+$ aq gefunden, die Kupferbase demnach selbst als einsäurig erkannt, und er hebt dies speciell hervor, indem er sagt: dass das Sättigungsvermögen der Base durch den Eintritt von $1 \ddot{\text { Aq}}$. Kupfer fur 1 Atom Wasserstoff auf die Hälfte herabgesetzt werde.

1 Ber. d. Deutschen chem. Gesellsch. 12, p. 781 u. 782. 
Bei der von mir ausgefuhrten Untersuchung des Äthylbiguanids hatte sich ergeben, dass diese leicht in grösserer Menge zu gewinnende Base (in ihrem kupferfreien Zustande) zwei Reihen von gut krystallisirten Salzen gibt, und zwar neutral reagirende, welche e in Äquivalent Säure enthielten und sauer reagirende, welche $z$ wei Äquivalente Säure enthielten, die man daher passend als neutrale und saure Salze bezeichnen konnte. Wenn man z. B. dem sauren schwefelsauren Äthylbiguanid $\mathrm{C}_{2} \mathrm{H}_{6}\left(\mathrm{C}_{2} \mathrm{H}_{5}\right) \mathrm{N}_{5} . \mathrm{H}_{2} \mathrm{SO}_{4}$ mit Barytwasser die Hälfte, also ein $\ddot{\mathrm{A}} q$. Schwefelsäure entzieht, so erhält man eine neutrale Flüssigkeit, aus der dann ein Äthylbiguanid mit halb soviel Schwefelsäure krystallisirt.

Nach diesem war es von vorne herein unwahrscheinlich, dass das einfache Biguanid eine andere Acidität besitzen sollte, als die äthylirte Base, es drängte sich vielmehr die Überzeugung. auf, dass auch das einfache Biguanid zwei Reihen von Salzen gibt, neutrale und saure, und dass das von $R$ ath $k$ e beschriebene Sulfat in Folge seiner Darstellung nur das saure Sulfat sein konnte. Rathke löste nämlich die im Wasser nicht lösliche schwefelsaure Kupferbase in verdunnter Schwefelsäure, entkupferte mit Schwefelwasserstoff und engte die nun überschitussige Schwefelsäure enthaltende Flïssigkeit ein, aus der dann das saure Sulfat auskrystallisiren musste. Die Zusammensetzung ist richtig angegeben worden, aber Rathke's Angabe, dass die Base ein Molekul Schwefelsäure zu n eutral reagirenden Salzen binde, ist unrichtig, denn dieses Salz reagirt stark saner, wie ich mich an einem von mir dargestellten Präparate leicht überzeugen konnte.

Dass sich diese Unrichtigkeit in Rathke's sonst so schöne Arbeit einschleichen konnte, erklärt sich aus dem Umstande, dass er gezwungen war, seine Untersuchungen mit ausserordentlich geringen Substanzmengen auszuführen.

Es war daher noch nöthig, das bisher nicht beschriebene n e utrale Biguanidsulfat darzustellen. Zu diesem Zwecke wurde nach Herth's Methode ' gewonnenes Biguanidkupfer mitSchwefelsäure genau neutralisirt, das so erhaltene Biguanidkupfersulfat

1 Monatshefte f. Chemie 1, p. 94. 
in Wasser suspendirt und durch Einleiten von Schwefelwasserstoff zerlegt. Das durch Abfiltriren vom Schwefelkupfer erhaltene Filtrat gab, zum Krystallisiren eingedampft, ein Salz, das nach seiner Reaction auf Lackmus und nach seiner Zusammensetzung als das eigentliche, neutrale schwefelsaure Biguanid $\left(\mathrm{C}_{2} \mathrm{H}_{7} \mathrm{~N}_{5}\right)_{2} \cdot \mathrm{H}_{2} \mathrm{SO}_{4}+2 \mathrm{H}_{2} \mathrm{O}$ bezeichnet werden muss.

\section{Analysen.}

1) $0.1132 \mathrm{Grm}$. krystallisirte Substanz gaben beim Verbrennen mit Kupferoxid and Bleichromat $42.5 \mathrm{CC}$. Stickstoff bei $21^{\circ} \mathrm{C}$. und $724 \mathrm{Mm}$. Druck.

2) $0 \cdot 1674$ Grm. krystallisirtes Salz gaben $0 \cdot 115 \mathrm{Grm} . \mathrm{Ba} \mathrm{SO}_{4}$.

3) Beim Trocknen bei $105^{\circ}$ erlitten $0.9417 \mathrm{Grm}$. einen Gewichtsverlust von $0 \cdot 1013 \mathrm{Grm}$.

Oder in 100 Theilen:

\begin{tabular}{|c|c|}
\hline $\begin{array}{c}\text { Berechnet für } \\
\left(\mathrm{C}_{2} \mathrm{H}_{7} \mathrm{~N}_{5}\right)_{2} \cdot \mathrm{H}_{2} \mathrm{SO}_{4}+2 \mathrm{H}_{2} \mathrm{O}\end{array}$ & Gefunden \\
\hline Stickstoff $\ldots \ldots \ldots 41 \cdot 7$ & $41 \cdot 8$ \\
\hline Schwefels. $\left(\mathrm{SO}_{3}\right) \cdot 23 \cdot 8$ & $23 \cdot 6$ \\
\hline Wasser........10.71 & $10 \cdot 76$ \\
\hline
\end{tabular}

Das neutrale schwefelsaure Biguanid stellt grosse, farblose Krystalle dar, die in Wasser sehr leicht löslich sind und bitterlich salzig schmecken; sie enthalten zwei Molekttle Krystallwasser.

Zur weiteren Controlle wurde nach Herth's Vorschrift dargestelltes, also saures Biguanidsulfat mit Barytwasser von bekanntem Gehalt bis zum Eintreten der neutralen Reaction titrirt; die vom $\mathrm{Ba} \mathrm{SO}_{4}$ abfiltrirte Flüssigkeit lieferte beim Eindampfen schöne Krystalle des neutralen Salzes (welche zur Analyse 2 gedient haben).

$0 \cdot 4325$ Grm. saures Biguanidsulfat $\mathrm{C}_{2} \mathrm{H}_{7} \mathrm{~N}_{5} \cdot \mathrm{H}_{2} \mathrm{SO}_{4}+\mathrm{H}_{2} \mathrm{O}$ verbrauchten $4 \cdot 35 \mathrm{CC}$. Barytwasser $\left(\dot{\mathbf{a}} 1 \mathrm{CC} .=18 \cdot 49 \mathrm{Mgrm} . \mathrm{SO}_{3}\right.$ ), diesen entsprechen $80.43 \mathrm{Mgrm} . \mathrm{SO}_{3}$ oder $18.6 \%$ vom Gewichte der angewandten Verbindung. Die Rechnung verlangt für $1 / 2$ Äq. $\mathrm{SO}_{3} 18 \cdot 5 \%$. 
Es ist sonach das Biguanid gleich seiner Kupferverbindung als eine einsäurige Base zu bezeichnen, da erst zwei Moleküle derselben ein Molekul der zweibasischen Schwefelsäure zur neutral en Flüssigkeit sättigen.

II.

Nach Ossikorszky ${ }^{1}$ spaltet sich das Guanidin beim anhaltenden Kochen mit Laugen oder Säuren unter Wasseraufnahme in Kohlensäure und Ammoniak, gemäss der Gleichung

$$
\mathrm{C}=\mathrm{NH}+2 \mathrm{H}_{2} \mathrm{O}=\mathrm{CO}_{2}+3 \mathrm{NH}_{3},
$$

welche jedoch nicht quantitativ festgestelit, sondern nur aus der Beobachtung erschlossen wurde, dass die Base beim Kochen mit Barytwasser Ammoniak, beim Erhitzen mit Säuren aber Kohlensäure entwickelte.

Wenn nun das Biguanid eine dem Guanidin analoge Constitution besitzt, so war es nicht unwahrscheinlich, dass es sich durch Einwirkung starker, Säuren und hoher Temperatur auch in ähnlicher Art, also nach dem Schema

$$
{ }_{\mathrm{CH}+4 \mathrm{H}_{2} \mathrm{O}=2 \mathrm{CO}_{2}+5 \mathrm{NH}_{3}}^{\mathrm{NH}=\mathrm{NH}}
$$

zersetzen liesse.

Um die Reaction quantitativ verfolgen zu können, wurden: gewogene Mengen sauren schwefelsauren Biguanids und titrirte Schwefelsäure von 1.47 spec. Gew. in ein Kaliglasrohr eingeschlossen und unter mehrmaligem öffnen successive bis auf

1 Ber. d. Deutschen chem. Gesellsch. 5, pag. 668. 
200 bis $210^{\circ}$ so lange erhitzt, bis eine Zunahme des innern Druckes nicht mehr beobachtet wurde. Die Menge gebildeten Ammoniaks wurde nach dem Öffnen des Rohres durch Titriren bestimmt.

1. Versuch. $\mathbf{0} 426$ Grm. krystallisirtes saures Biguanidsulfat $\left(\mathrm{C}_{2} \mathrm{H}_{7} \mathrm{~N}_{5} \cdot \mathrm{H}_{2} \mathrm{SO}_{4}+\mathrm{H}_{2} \mathrm{O}\right)$ wurden mit $6 \cdot 7135$ Grm. eines Gemisches von 1 Vol. concentrirter Schwefelsäure mit 1 Vol. Wasser [1 Grm. dieser verdünnten Säure entsprach $42 \cdot 9$ CC. Lauge (à 1 CC. $=10 \cdot 88 \mathrm{Mgrm} . \mathrm{Na} \mathrm{OH}$ )] etwa 6 Stunden auf $170^{\circ}$ und 12 Stunden auf $200-210^{\circ}$ erhitzt. Zur Neutralisation von 25 CC. des auf 250 CC. verdünnten, wasserklaren Röhreninhaltes waren $26 \cdot 6$ CC. Lauge erforderlich. ${ }^{1}$

2. Versuch. $0 \cdot 2743$ Grm. derselben Substanz wurden mit $2 \cdot 7595$ Grm. der obigen verdïnnten Säure wie unter 1) erhitzt. Der Röhreninhalt wurde auf $100 \mathrm{CC}$. verdiunnt und zur Nentralisation von $50 \mathrm{CC} .52 \cdot 7 \mathrm{CC}$. Lauge gebraucht.

Zum Überflusse wurde das entstandene Ammoniak in beiden Fällen in einer kleinen Probe qualitativ nachgewiesen.

In der folgenden Übersicht sind die Resultate der beiden Versuche zusammengestellt:

\begin{tabular}{|c|c|c|}
\hline & Versuch 1. & Versuch 2. \\
\hline $\begin{array}{l}\text { Der angewandten Schwefel- } \\
\text { säure entsprachen........ } \\
\text { der Schwefelsäure des sauren } \\
\text { Biguanidsulfats .......... }\end{array}$ & $288 \cdot 0$ CC. Lauge & $118 \cdot 4$ CC. Lauge \\
\hline $\begin{array}{c}\text { in Summe.. } \\
\text { zum Rücktitriren wurden } \\
\text { verbraucht ........... }\end{array}$ & $\begin{array}{l}302 \cdot 5 \text { CC. Lauge } \\
266 \cdot 0 \quad, \quad,\end{array}$ & $\begin{array}{l}127 \cdot 7 \text { CC. Lauge } \\
105 \cdot 4 n, n\end{array}$ \\
\hline $\begin{array}{l}\text { dem entstandenen } \mathrm{NH}_{3} \text { ent- } \\
\text { sprachen demnach } \ldots \ldots \ldots \\
\text { d. h. } \\
\text { gebildetes Ammoniak.. } \\
\text { (berechnet ............ }\end{array}$ & $\begin{array}{l}36 \cdot 5 \text { CC. Lauge } \\
169 \text { Mgrm. } \mathrm{NH}_{3} \\
167 \quad n \quad n\end{array}$ & $\begin{array}{l}22 \cdot 3 \text { CC. Lauge } \\
103 \mathrm{Mgrm} . \mathrm{NH}_{3} \\
107 \quad " \quad, \quad)\end{array}$ \\
\hline
\end{tabular}

1 Die von der Schwefelsäure dem Glase entnommene Alkalimenge konnte vernachlässigt werden, da sie, wie ich mich durch Eindampfen eines gemessenen Flïssigkeitsquantums ïberzeugte, nur etwa $2 \mathrm{Mgrm} . \mathrm{NH}_{3}$ entsprach. 
Durch diese Zahlen ist festgestellt, dass sich das Biguanid beim Erhitzen mit Schwefelsäure von 1.47 spec. Gew, unter Wasseraufnahme glatt in Kohlensäure und Ammoniak spaltet, und es gewinnt hiedurch die Annahme der Constitution, die jener des Guanidins analog ist, sicher neuerdings an Wahrscheinlichkeit.

Es kann noch beigefügt werden, dass ein paar Versuche, Biguanid durch Einwirkung heisser, concentrirter Ammoniakflüssigkeit in Guanidin überzuführen, zu keinem positiven Resultate führten.

Graz, im April 1883. 